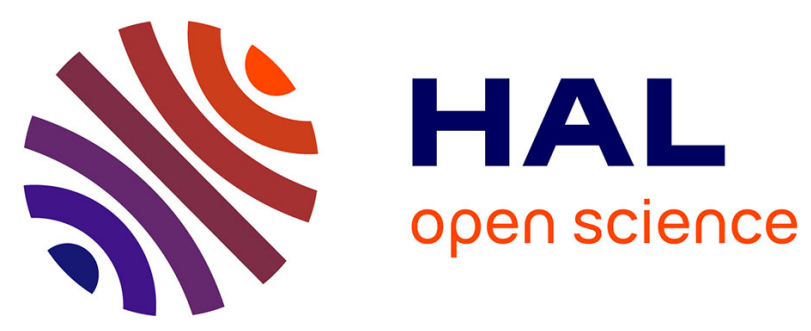

\title{
À propos du milliaire de Cneus Domitius Ahenobarbus trouvé dans l'Aude en 1949 \\ Paul-Marie Duval
}

\section{To cite this version:}

Paul-Marie Duval. À propos du milliaire de Cneus Domitius Ahenobarbus trouvé dans l'Aude en 1949. Gallia - Fouilles et monuments archéologiques en France métropolitaine, 1949, 7 (2), pp.207231. 10.3406/galia.1949.2134 . hal-01920423

\section{HAL Id: hal-01920423 \\ https://hal.science/hal-01920423}

Submitted on 27 Feb 2020

HAL is a multi-disciplinary open access archive for the deposit and dissemination of scientific research documents, whether they are published or not. The documents may come from teaching and research institutions in France or abroad, or from public or private research centers.
L'archive ouverte pluridisciplinaire HAL, est destinée au dépôt et à la diffusion de documents scientifiques de niveau recherche, publiés ou non, émanant des établissements d'enseignement et de recherche français ou étrangers, des laboratoires publics ou privés.

\section{(ㅇ)(1) $\$$}

Distributed under a Creative Commons Attribution - NonCommercial - NoDerivatives| 4.0 


\title{
A PROPOS DU MILLIAIRE DE CNEUS DOMITIUS AHENOBARBUS TROUVÉ DANS L'AUDE EN 1949.
}

\author{
par M. Paul-Marie Duval
}

L'intérêt de cette découverte n'échappera à personne. M. Joseph Campardou, professeur à la Faculté des Sciences de Toulouse, a bien voulu confier à Gallia une étude de ce document, qui a déjà fait l'objet d'une communication de lui-même à la Société archéologique du Midi de la France (inédite) et d'une communication de M. Philippe Héléna à la Commission archéologique de Narbonne (Bulletin, XXII, 2, pp. 88-102). La pierre a été examinés par M. Jean Jannoray, Directeur de la XI circonscription des Antiquités historiques, et l'inscription soigneusement relevée : frottis à la mine de plomb, estampage et photographies en confirment la lecture, indiscutable malgré la forte érosion du grès. La borne a été protégée par les alluvions du torrent, l'inscription est ancienne et n'a jamais été retouchée (v. plus haut, p. 195 sq.).

Le lieu de découverte pose un problème à la solution duquel M. Campardou, fort de ses études passées sur la voie Domitienne au sud de Narbonne, s'est particulièrement attaché. Il s'était déjà convaincu du passage dans la plaine de la voie préhistorique des sauniers du littoral, voie légendaire d'Héraklès, voie historique d'Hannibal. La découverte du milliaire impose sa conviction : c'est ce tracé qu'a emprunté la première voie Domitienne. La borne est en effet trop lourde pour avoir été roulée sur $800^{\mathrm{m}}$ par le Rieu depuis un gué supérieur où passerait une voie empruntant les collines, et le chiffre de $\mathrm{XX}$ milles correspond à la distance de Narbonne en ligne presque droite. D'ailleurs cette découverte, provoquant un examen plus attentif du terrain, a conduit M. Campardou à reconnaître les restes d'un gué aménagé dans l'antiquité à proximité immédiate du milliaire basculé. Tout cela confirme l'existence de la voie primitive du littoral. Le détail du tracé des voies et de leurs variantes successives jusqu'au Bas-Empire, établi d'après les autres découvertes, l'étude du terrain et la toponymie, reste l'apport personnel des tra. vaux de M. Campardou. 
Le nom que porte le milliaire, suivi du mot imperator, l'aspect du monument, les caractères et le texte de l'inscription nous reportent à l'époque républicaine, et singulièrement au premières années de la présence romaine en Gaule.

Ce Cneus Domitius Ahenobarbus, fils de Cneus, et imperator, ne peut être que le consul de 122, le vainqueur des Allobroges et des Arvernes. De toute la lignée, il est le seul qui ait, à la fois, partagé avec son père le prénom de Cneus et mérité la dignité d'imperator ${ }^{1}$, après avoir accédé au consulat.

Un milliaire du II $^{\mathbf{e}}$ siècle avant J.-G. est chose rare, mais non exceptionnelle. Sans doute, le petit nombre des milliaires connus de la République est surprenant ${ }^{2}$ : échelonnés principalement de 187 à 75 environ, ils sont au nombre d'une quinzaine pour l'Italie, d'une douzaine pour les provinces. Mais l'on sait par Plutarque que Caius Gracchus a le premier généralisé leur emploi (lequel remonte au III $^{\ominus}$ siècle); et c'est en 129 qu'apparaissent les premiers milliaires connus dans les provinces ${ }^{3}$. Or, si le milliaire de Domitius est le premier milliaire républicain connu en Gaule, il remonte précisément à l'époque de Caius Gracchus, qui ne paraît pas avoir été défavorable à la nouvelle conquête $^{*}$ : et c'est ce qui importe ${ }^{5}$. Le petit nombre ou l'absence des décou.

(1) Voir la généalogie dans R.-E., $\mathrm{V}(1905)$, s. v. Domitius, col. 1315 (MüNZER) et dans DrvMnN-Grosne, Geschichte Roms etc..., III (1906), pp. 13-18. De tous les Domitii, depuis l'ancêtre Lucius jusqu'au dernier Lucius (l'empereur Néron), 4 seulement sont Cneus, fils de Cneus: le consul de 162, son fils consul en 122, l'un des fils de celui-ci, consul en 96 (après avoir été fondateur de Narbonne en 117, v. plus loin n. 34), et le fils de ce dernier; et un seul a mérité le titre d'imperator, c'est l'un des trois qui ont accédé au consulat, celui dont le triomple est mentionnó dans les «Fasles triomphaux 》: c'est le consul de 122, le vainqueur de la Gaule méridionale. Nous ne savons pas en effet si son fils Cneus a jamais été appelé imperator. S'il a reçu les honneurs du triomphe, cela ne pourrait ètre quavant son consulat de 96 , entre 103 et 99 , seules années pour lesquelles la liste des Fasti comporte une lacune. Nous ne savons pas non plus s'il est relourné en Gaule après sa parlicipation a la fondation de Narbonne au début de sa carrière (v. plus loin, n. 34).

(2) Le volume du C. I. L. consacré aux milliaires n'est pas encore paıu. En attendant, il faut se reporter au mémoire de O. HIRscurbun, Die römischen Meilensteine (190\%), dans ses Kleine schriften, ch. XLIV, p. 703-i43. Environ deux douzaines de milliaires pour l'époque républicaine, dont 15 en Ilalie (contre quatre milliers pour l'époque impériale). La 2 édilion du C.l.L., I consacré a l'époque républicaine (I, 21, 1918,22 1934, 23 1943) n'en ajoute pas de nouveaux, non plus que l'article, d'ailleurs très rapide, de H. U. INs'TrssiY, J.D.A.I., 56, 1941, col. 879-891.

(3) Ceux de $\mathrm{M}^{\prime}$ Aquilius en $\Lambda$ sie, v. plus loin, n. 14. Le milliaire de Domilius est le premier connu à l'Ouest de l'Italie avant l'śpoque impériale que l'on puisse dater, et peut-ètre le plus ancien: les trois milliaires de Tarraconnaise classés à l'époque républicaine ne sont pas datés (C.I.L., I, 21840 (1 re de. 1486)), $823(1894), 824(1485)=$ II 4956, 4924, 4925: le premier serait le plus ancien).

(4) J. Cancoprio, Histoire romaine de la coll. Glotz, II, p. 284: «alor's à l'apogée, Caius, oubliant les dissentiments du passé, ne marchanda point son concours à Domitius »(en 122).

(5) On a trouvé en Italie, sur la voie Appienne, un milliairc, de lecture incertaine, que l'on a parfois attribué (Instinsky, o. l.) au consul de 122:Cn. $D[0] m[i t i] u[s] /$ Cos. / CXII (C.I.L., I, 21 
vertes passíes peurent s'expliquer par la faible étendue dı monde rmmitin d'alors, par la rarelé des monuments du $\mathrm{It}^{a}$ siècle qui sont parvenus jusilüa nous, enfin par une exploration insuffisante: "le nombre des milliaires sonnus en Gaule était de cinq au début du xix siècle ", écrivait naguère M. . Albert Grenier, " on peut espérer encore de nouvelles trouvailles " ${ }^{6} .$. . I rai dire, il était curieux que la voie Domitienne, la plus ancienne et la plus rélèbre de la Gaule. n'eût pas encore liveé de milliaires antérieurs à l'Empiro.

L'aspect matériel du monument correspond à ce que nous savons des milliaires de l'époque républicaine. Deux d'entre eux, datés de 187, onl mème, à 2 ou $3^{\mathrm{cm}}$ près, les mèmes mesures ${ }^{7}$ : ils ont été, il est vrai, trourís en

322 (629) $=\mathrm{X} 6872)$ : le document est peu stir et le C.I.L. ne se prononce pas, pour la dill, entre les consuls de 192, 162, 192 el 96. Isil decouverte du milliaire de Domitius en Gaule ne rend en aucune façon ce document plus ralable et ce dernier ne nous est d'aucun secours pour litude du nouveau milliaire.

(6) Iranuel d'archéol. gallo-romaine, VI, 1, p. 53. En 1907, Hirschfeld, o. l., complail +"mirun 550 milliaires pour l'ensemble des Gaules et de la Germanie.

(7) Les mesures des mi'liaires de la République n'ont pas toujours été notées aver lunle la précision désirable. Les pierres sont parfois cassées, ou enterrées en partic, et dans le seronul cas on ne signale pas si la mesure est complete. Voici les renseignements les mieux assurés, dapris le C.I.L. (pour le t. I, nous citerons la $2^{2}$ édition en tète, et entre parenthèses la $1^{\text {re }}$ ):

\begin{tabular}{|c|c|c|c|c|c|}
\hline C.I.L. I, 21 & Signataire & Forme & Iauteur & Diamètre & Dille: \\
\hline $21=X 6838$ & $\begin{array}{l}\text { P. Claudius } \\
\text { A (p. f.) ef } \\
\text { (.. Focrius, } \\
\text { édiles }\end{array}$ & colonile & $0 \mathrm{m.} .78$ & $0 \mathrm{~m} .61$ & avall $: 19$ \\
\hline $229(6.33)=\mathrm{XI} 6616$ & $\begin{array}{l}\text { [). MENATES, } \\
\text { édile }\end{array}$ & (volonne & & $\begin{array}{l}3 \text { palmes }= \\
\text { env. } 0 \mathrm{~m} .68\end{array}$ & 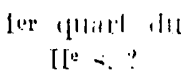 \\
\hline$(519(53 \pi)=X[6641$ & $\begin{array}{l}\text { M. ENLLILS } \\
\text { I.EPIDUS }\end{array}$ & rul. rassée & $1 \mathrm{~m} .15$ & $\begin{array}{l}\text { I m. } \$ 6(?) \\
\text { en liaut }\end{array}$ & IN \\
\hline $1517(535)=$ XI 6642 & $\gg$ & $\begin{array}{l}\text { col. conique } \\
\text { a base carré } \\
\text { lalge de } 0,6 ?\end{array}$ & $\begin{array}{cc}1 \mathrm{~m} . & 30 \\
\text { suns } & \text { la base }\end{array}$ & $\begin{array}{l}\text { () } \mathrm{m} .44 \\
\text { en llaut }\end{array}$ & 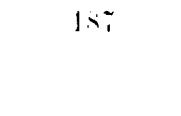 \\
\hline $618(536)=$ XI 6645 & $D$ & «il. » & «ill.» & 《id.» & 127 \\
\hline $63 \%(550\rangle=V 8007$ & $\begin{array}{l}\text { POPIIJIUS } \\
\text { LAENAS }\end{array}$ & poinlu en bas & $1 \mathrm{~m} .22$ & $0 \mathrm{~m} .65$ & 132 \\
\hline
\end{tabular}

On voit que le $\dot{4}^{\mathrm{e}}$ et te $5^{\mathrm{e}}$ milliaires de celte lisle $(617$ et le suivant que le C.I.L. donue me semblable a lui) doivent itre hauts de $1 \mathrm{~m} .30$, + env. $0 \mathrm{~m} .60$ pour la base "carrée "s soit l m. 90 env., et qu'avec leur diam. de $0 \mathrm{~m}$. $\mathbf{i} \mathbf{i}$ en haut, ils sont très comparables au milliaire de Domitius (h. $1 \mathrm{~m} .93$, diam. $0 \mathrm{~m}$. 45 en haut, $0 \mathrm{~m}$. 43 en bas): les seules différences sont lexislence d'une base, que ce dernier ne comporte pas, et la forme, conique et non cylindrique. Pour les autres, par le mot "colonne», seul, le C.I.L. indique très vraisemblablement la forme rylinarique). 
Italie; malheurcusement, nous n'avons pas de description détaillée des milliaires provinciaux ${ }^{8}$.

Les lettres ont une forme courante dans l'épigraphie des milliaires, et particulièrement à l'époque républicaine (fig. 1). Gravées non sur la surface plate d'un cartouche mais, comme sur d'autres milliaires de toutes époques (les moins soignés), sur la courbe même de la borne dont elles couvrent les $4 / 5$ au niveau de la $1^{\text {ro }}$ ligne, elles présentent une disposition irrégulière qui est dûe à la difficulté de graver dans ces conditions sur un bloc de cette taille. péniblement maniable. On serait tenté d'attribuer à cette difficulté technique le fait que certains caractères ont une allure cursive (les $G$ très ouverts, les $M$ un peu couchés, le $\mathrm{V}$ ventru de Ahenobarbus): mais on retrouve cette particularité sur des inscriptions du $\mathrm{II}^{\mathrm{e}}$ siècle gravées sur surface plate, notamment sur l'ex-voto de Mummius, daté de 146, dont l'alphabet présente de grandes analogies avec celui du milliaire, nolamment pour le mot imperator ${ }^{10}$. Mais les analogies les plus probantes sont fournies par les inscriptions de milliaires ou de cippes : milliaires de M. Emilius Lepidus en 187, de S. Postumius Albinus en 148, de L. Cornelius Cina en 127, de T. Quinctius Flamininus en 127-123(?), et surtout cippes gracchiens de 130-121 où se retrouvent le même alphabet et la même disposition irrégulière ${ }^{11}$. On notera le fait que trois lettres se présentent chacune sous deux formes différentes sur le milliaire: mais on a des exemples de ce fait et des mêmes formes à la même époque, et. qui plus est, dans des inscriptions sur fond plat ${ }^{12}$ : c'est sans doute le seul

(8) Les milliaires anépigraphes de Ponl-de-Gart (voie Domitienne), hauts de $2 \mathrm{~m} .40$ et $2 \mathrm{~m} .28$ (diam. $0 \mathrm{~m}$. 74), sont très probablement du Haut-Empire (C.I.L. XII, 5614-5615, $\Lambda$. Grexikr, Manuel, VI, 1, 1934, p. 45, n. 2) comme ceux avec lesquels ils ont été trouvés, hauts de $2 \mathrm{~m}$. 78 (XII, 5613, $\Lambda$ uguste), $2 \mathrm{~m}$. 68 et $2 \mathrm{~m} .97$ (5612 et 5611, Glaude). D'une façon générale, les milliaires impériaux sont plus hauts, sauf au Bas-Empire, que ceux que l'on connait de la République (ibid., p. 69-71), tous inférieurs à 2 m., comme le milliaire de Domitius.

(9) Longue de $1 \mathrm{~m} .12$, pour une circonférence de $1 \mathrm{~m} .41$.

(10) C.I.L., I, 21626 (541) = III, 331, photo dans R. CaG.Mat, Cours d'épigraphie latine, $4^{\mathrm{e}}$ éd., 1914, pl. III, $\mathrm{n}^{\circ}$ 4, froltis reproduit dans Rirschl, Priscae Latinitatis Monumenta Epigraphica, 18\%2. pl. LI, A. Voir aussi l'inscription de Popillius Laenas, consul en 132 , relative a la création et au bornage de la Via Popillia (I, $21638(551)=\mathrm{X}, 6950$; GaG.Nat, o. l., pl. II, nº 2, Ritschl, pl. LI, B).

(11) Références plus loin, n. 14. Reproduction des froltis dans Ritscirl, o. $l$., pl. XLVIII A (Lepidus), LV $\Lambda$ (Postumius), LVI $\Lambda$ (Cina), LVI B (Flamininus), LV $\mathrm{G}$ et $\mathrm{n}$ et XCVIII $\Lambda$ (cippes gracchiens). L'inscription du milliaire de Popillius Laenas, érigé en 132 (v. plus haut, n. 7) est en beaux caractìres gravess sur une surface plate (CagNat, o. l., pl. II, no 1 ). Celles des milliaires du III siècle (C.I.L., I, 2121 (= X 6838) et 22 (= VI 1277 et 31.585, dessins) sont franchement archaïques. M. J. Carcopino a attiré mon attention sur les cippes gracchiens: qu'il veuille bien trouver ici mes remerciements.

(12) Le B, dans Ahenobarbus (le ter a la boucle supérieure plus petite, le $2^{\mathrm{e}}$ a les deux boucles égales), le $\mathrm{R}$ dans imperator et dans $A$. (le 1er de $i$. a le jambage latéral convexe, les autres l'ont rectiligne), le $\mathrm{V}$ dans $A$. et le autres mots (ventru dans le 1er, rectiligne ailleurs). Voir C.I.L. I, 21 614, en 189 (les deux B, $3^{\mathrm{e}}$ et $4^{\mathrm{e}}$ lignes, photo); 615 à la mème époque (les deux 


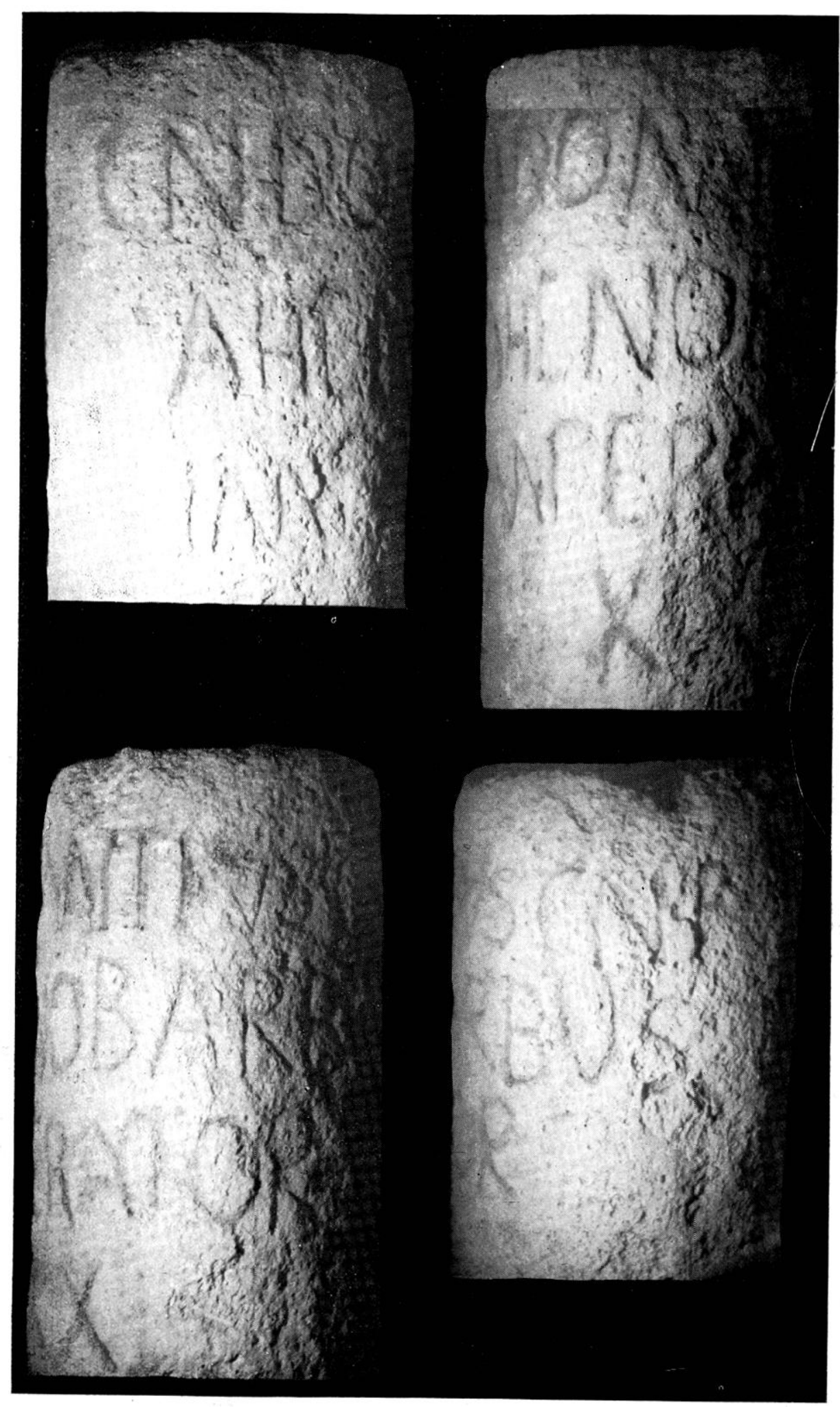

Cl. J. Campardou.

Fig. 1. - Le milliaire de Domitius. Partie supérieure, portant l'inscription : CN.DOMITIVS CN.F. / AHENOBARBVS / IMPERATOR / XX (Hauteur des lettres : 10 à $12 \mathrm{~cm}$.) 
indice d' "archaïsme" que l'on pourrait déceler dans cette inscription, encore qu'on puisse mettre cetle irrégularité sur le compte de la maladresse dont témoigne la gravure. Plus encore qu'à la surface courbe de ce cylindre au faible diamètre, c'est à la mauvaise qualité de la pierre qu'il faut attribuer la principale différence qui distingue cette inscription provinciale des inscriptions de même nature et de même époque qu'on connaît en Italie : celles-ci, grarćes sur tuf, sont plus profondes et plus nettes que la nôtre, gravée sur un grès tendre qui, par surcroît, est aujourd'hui fortement émoussé. Cette grarure légère n'est pas le fait d'un âge plus récent, mais des difficultés direrses auxquelles son auteur a dû faire front.

Enfin, l'orthographe n'offre rien que de normal ${ }^{13}$. En bref, nous avons ¿ faire à une inscription peu soignée, témoin d'un travail difficile et de natur' strictement utilitaire, et qui est parfaitement à sa place dans le matériel épigraphique du $\mathrm{Ir}^{\mathrm{e}}$ siècle avant notre ère.

La formule, dans sa concision, correspond elle aussi à celle des milliaires de la République qui parfois, d'ailleurs, portent seulement le nombre des millis: prénom, nom, filiation indiquée par le prénom du père, surnom, titre (édile au III $^{\mathrm{e}}$ siècle, consul ou proconsul ensuite), - distance ${ }^{14}$. Le mot imperator ne se trouve sur aucun milliaire connu avant l'extrême fin de l'époque républicaine, et, sur ces documents de l'année 28, il accompagne le titre de consul ${ }^{15}$, tandis que sur le milliaire de Domitius, il est seul mentionné. Mais nous savons par bien d'autres documents que ce choix a été d'usage

$\mathrm{R}$, phulo); voir aussi 692 a la fin du II siècle (B à boucles inégales, photo), 30 a l'époque la plus ancienne de Rome ( $\mathrm{R}$ a jambage courbé; 2 fois,pholo); -382 la plus ancienne inscription du Picenum (sur patère: les de $u x \mathrm{~V}$, fig.).

(13) Les noms propres n'offrent point ici de prise à l'étude. Pour imperalor, on connaît sur les inscriptions des formes archaïques: impeirator en 189 en Espagne (C.I.L., I, $21614=$ II, 5041 , inperator en 167 en Gièce (I, $21622=I I I, 14203$, no 22), mais on a déjà la forme classique en 146 (L. Mummius, imperator, v. plus haut, n. 10).

(14) On trouve seulement parfois, en plus, la filiation indiquée par le prénom du grand-père, le nom de la ville chois:e comme point de départ ou point d'arrivée, et un ou deux chiffres supplémentaires relatifs à d'autres villes. Voici des exemples datés (nous laissons de côté les indications de distances): en 187, M. Emiluus, M. f., M. n. / Lepidus. Cos (C.I.L., I, 21617 (535)

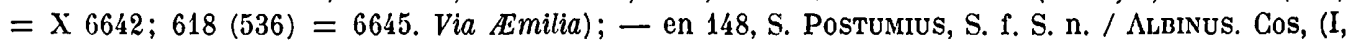
$21624(540)=$ V 8045. Via Postumia); - en 132, P. Popilluus, C. f. / Cos. (I, $21637(530)=$ V 800 i). Voie d'Ariminum à Aquiléc); - en 129, M'AQuillius, M'. f. / Cos. (I, $21647=$ III 6093 et. suppl. 7183, entre cutres, rédigcées en latin et en grec : I, $21646=651$. Via Aquillia, en Asie); - en $12 \%$, L. Connelius, L. (f.) Cina, Cos (I, 21654 (558) $=$ X 6905. Entre Venafrum et $\mathrm{Na}$ ples:; - en 123 (?), T. Quinctius, T. f. Flamininus / Cos, (I, 21657 (559) = XI 6671); - ver's 115 (?), L. CAecili, Q. f. / Metel. Cos. (I, 21661 (561) = IX 5953. Via Caecilia, probabliment); en 74. P. Serveilius, P. (f.) / Isauricus Procos. / res(tit)uit (I, 21786 (622) = III, 462. En Asie); - ell 28, C. Calvisius, G. f. Sabinus. Gos. / Imp. (X 6895, 6897, 6899, 6901. Près d'Aquinum), etc...

(15) Voir à la fin de la note précédente, milliaires de G. Calvisius. Cette habitude, pour un consul, de porter son titre d'imperator, appartient à la fin de la République, v. note suiv. 
jusqu'à l'époque de César : le titre suprême, à partir du moment où il a été décerné par les troupes, était, le plus souvent, seul adopté par son bénéficiaire, éclipsant notamment le titre proconsulaire. On le trouve ainsi, seul, accolé au nom de l'auteur d'un document officiel ou d'une dédicace à partir de $189^{16}$, et couramment accolé au nom d'un monétaire à partir de $82^{17}$. Or, le bornage d'une voie, même neuve, n'est pas un acte plus officiel que la promulgation d'un décret proconsulaire, la dédicace d'un monument public, ou l'émission d'une série monétaire : il n'y a donc rien que de très normal à trouver le titre d'imperator sur un milliaire, et à l'y trouver seul, -... ce serait la présence du titre proconsulaire à côté de lui qui serait insolite ${ }^{18}$.

Enfin, le fait qu'un magistrat, ouvrant une voie nouvelle, la marque à son nom en portant celui-ci sur ses milliaires est, lui aussi, tout à fait normal. Le cas de M' Aquillius, chargé en 129 d'organiser la nouvelle province d'Asie, est significatif à cet égard : il y aménage la route de Sardes à la romaine en y posant ses milliaires, et ce sera la Via Aquillia ${ }^{19}$. Un document

(16) Momssix, Droit public, t. I (1887) p. 143-149; ce titre est une distinetion qui serl d'acheminement au triomphe, il éclipse tous les autres à partir du moment où il a êté décerné par les troupes. Rosexbeng (art. imperator dans R. E. IX (1916), col. 1141-1142) nuance ainsi la doctrine: il n'est pas d'usage de porter à la fois le titre d'i. et celui de consul (on ne trouve les deux que dans le rappel chronologique d'une tilulature: L. Mummius ... cos ... dedit, Romam rediit triumphans..., quod in bello vocerat ... imperator dedicat (C.I.L., I, 21626 (5.11) = VI 331, en 145); Ap. Claudius Pulcher ... consul vovit..., imperator coepit $(\mathrm{I}, 21$ \%55 $(619)=$ III 547, en 48$)$ ou celui de dictateur (Sulla préfère ce dernier acquis en 81 , à celui d'i. acquis dès la fin de la guerre contre Mithridate). De plus, les inscriptions du II ${ }^{\text {e }}$ siècle montrent que le consul, acclamé $i$. pendant son consulat, continue a porter son titre consulaire de préférence au litre nouveau jusqu'd la fin de son consulat, tandis que les magistrats prorogés, gouverneurs de province, proconsuls, portent de préférence le litre d'i. (tendance qui s'affirme jusqu'à César où ces distinctions disparaissent puisqu'il garde officiellement le titre d'i. après ses triomphes: d'où, à la fin de la République, en 28, le cons. imp. de C. Calvisius, v. plus haut, n. 14, à la fin): c'est, précisément, le cas de Domitius, qui était déjà proconsul quand il a cu la possibilité, la guerre terminée, de créer la voie d'Espagne. Pour les exemples, v. plus loin, n. 28.

(17) A partir de Sulla, des monétaires font suivre leur nom du titre d'i., généralement seul, aussi bien dans les provinces qu’à Rome: v. C.I.L., I, 22, Appendix nummorum, $28 \mathrm{i} \mathrm{sq}$.; H. A. Grueber, Coins of the Roman Republic in the British II useum, 1910, I, p. 359, n० 2895, p. 4i5, $n^{\circ} 3837$ (Rome); II p. 459, no 1 (Orient), p. 357, n० 47 (Espagne); pour la Gaule: II, p. $388, n^{\circ} 1$, C. Val. Fla(ccus) imperator (C.I.L. I, 22287 ), en 82 ; $i$, avant 83 , il n'a triomphé qu'en 81 , ex Celtiberia ... et Gallia. Sur les monnaies, le titre est presque toujours seul. Voir d'autres exemples, plus loin, n. 28.

(18) Point n'est besoin d'invoquer ici l'orgueil bien connu du personnage pour justifier l'emploi du titre d'i. de préférence à celui de proconsul, encore que l'indication psychologique ne soit point négligeable. - On pourrait, d'autre part, objecter que, sur les milliaires, l'indication de la magistrature est nécessaire parce qu'elle a valeur de date et que ces documents administratifs sont ainsi souvent datés: mais de nombreux milliaires n'offrenl que le chifr'e des milles pour toute indication, et le mot imperator accolé au nom de Domitius, rappelant la conquête mème du pays, tenait lieu de date approximative.

(19) V. plus haut, n. 14, avec d'autres exemples: milliaires de M. Emilius Lepidus sur la Via Emilia, de S. Postumius Albinus sur la Via Postumia: une route apparait ainsi comme la propriété morale d'une famille, à qui son entretien est à cœur, en lui fournissant un moyen de perpétuer son nom. 
nous est ici plus précieux que tout autre : l'inscription de P. Popillius Laenas qui, en 132, rappelle qu'il a créé la voie de Rhegium à Capoue, qu'il y a fait des ponts et posé des milliaires et des tabellarii (c'est-à-dire, probablement, des bornes intermédiaires) ${ }^{20}$; connaissant, sur une autre voie d'Italie, un milliaire à son nom ${ }^{21}$, on ne saurait penser que ce nom fût absent de la voie dont Popillius réclamait la paternité, et que l'on appellera la ria Popillia. Ainsi, quelques années avant le temps de Domitius et probablement depuis longlemps déjà, cet usage était bien ítabli. La seule nouveauté du milliairı de Domitius, c'est que l'auteur de la voie soit le conquérant lui-même imperator - du pays qu'elle traverse: c'est là sa valeur singulière.

Le milliaire de Treilles peut donc être attribué à Domitius en toute sé-. curité. Ce n'est pas seulement le premier milliaire de la République trouvé en Gaule, et l'un des rares connus dans le monde romain. G'est aussi la plus ancienne inscription latine découverte en territoire gallo-romain ${ }^{22}$, ct l'on nc pourrait guère en trouver de plus ancienne car elle témoigne de l'introduction même du nom latin sur le sol de la Gaule : dans sa rude majesté, cette borne marque un tournant décisif de notre histoire nationale. Témoin isolé d'une auvre d'ensemble - le bornage de la plus vieille route des Gaules elle est enfin la preuve que cette Via Domitia, dont nous ne connaissions pas le nom avant l'époque de Cicéron ${ }^{23}$, a bien été construite par le conquérant lui-même, - auquel on avait, d'ailleurs, toujours attribué la fondation sur son parcours d'un Forum Domitii ${ }^{24}$ : ce qui ćtait hier conviction ${ }^{25}$ esl alljourd'hui certitude.

(20) Pour l'inscription, v. plus haut. n. 10: sur les tabellarii, $\Lambda$. Grevien, Manuel, VI, 1, p. 80 sq., et. [H. G. PrLaum], Essai sur le cursus publicus sous le Ilaut-Empire romain, Mémoires présentés par divers savants à l'Académie des Inscript. et Belles-Lettres, XIV, $1^{\text {re }}$ partie, 1940, p. 242244.

(21) V. plus haut, n. 14 et 10.

(22) Jusque là, la plus ancienne inscription latine gravéc sur pierre en Gaule était, à Toulouse, une dédicace de l'époque de César, datée de 47 av. J.-C. (C.I.L., XII 5388).

(23) Iro Fonteio, VIII, 18 : cum majoribus reipublicae negotiis $M$. Fonteius impediretur, et cum ad rem publicam pertineret riam Domitiam muniri, legatis suis, primariis viris, C. Annio Bellieno et $C$. Fonteio, negotium dedit; itaque praefuerunt. On a parfois donné ici à munire le sens de "achever d'aménager», comme si la voie, vers 75, aprìs plus de 40 ans, n'était pas encore terminée. Mais la voie, qui porte un nom, est dejja faite : il est normal que l'on ait. besoin, au moment de la guerre d'Espagne, de renforcer son empierrement sur tout ou partie de son parcours. Dans la dernière traduction frangaise du Pro Fronteio ( $\Lambda$. Boulanger, Coll. des Univ. de France, t. VII des Diseour's de Cicéron), munitio viarum et munire sont constamment traduits, dans ce ch. VIII, par «la réfection des routes» et «travailler à 》 cette «réfection».

(24) V. plus bas, n. 68 .

(25) C. Julliax, Ilist. de la Gaule, III (1909), p. 36, n. 1: «le nom de la via Domitia n'apparait qu'au temps de Cicéron...: mais ce nom indique la date de la construction ». - M. BESNIER, art. via du Diction. des Antiq. (V, 1911, col. 799): "La plus ancienne voie romaine de la Gaule est la Via Domitia, qui date de l'établissement mème des conquérants en Narbonnaise ». - J. GAR- 
Peut-on préciser le moment de ses relations avec la Gaule où Domitius a marqué ainsi de son nom, en l'aménageant à la romaine, la vieille piste qui jusqu'alor's venait d'Espagne sans y conduire et sur laquelle il a le premier, moins de cent ans après Cannes et dans l'appareil mème qui avait fait trembler Rome ${ }^{26}$, reconduit le souvenir d'Hannibal jusqu'aux Pyrénées?

Lne certaine obscurité enveloppe ces quelques années, si importantes pour l'histoire des origines de la Gaule. Nos incertitudes commencent, en effet, avec l'arrivée même en Gaule de Domitius consul en 122, suivie en 121 par celle de Q. Maximus, son sucsesseur au consulat : on discute encore, sur la base fragile de textes rares, contradictoires et d'époques trop diverses, des rôles et des adversaires respectifs ou communs des deux généraux dans la campagne décisive qui livra aux Romains le nord de la vallée du Rhône par la défaite écrasante des Allobroges et de leurs alliés les Arvernes. Mais ce n'est pas le lieu de reprendre ici cette discussion épineuse ${ }^{27}$, car elle ne concerne pas directement le milliaire de Domitius : il faut le confronter avec ce que nous savons des événements qui ont suivi ces victoires.

Tout d'abord, quelles sont les exigences du document? II date du séjour en Gaule de Domitius imperator, et se place donc entre le moment où la conquête de la vallée du Rhône a valu le titre au proconsul et rendu possible l'ouverture de la voie d'Espagne, et celui où Domitius est rentré à Rome recevoir les honneurs du triomphe et déposer à tout jamais le titre suprême ${ }^{28}$ Entre ces deux moments, au moins les premiers mois, peut-être la premièrıs

copiso, o. l. (1929), p. 286 : «Domitius ... répare et assujettit à son contròle la vieille route qui... s'appellera dorénavant la Via Domitia». - A. Grexien, Manuel, VI, 1 (1934), p. 28 : «le nom seul de la route, Via Domilia, rappelant celui du proconsul de $121, \ldots$ reporte l'entreprise de la construction vers l'an 120 avant notre ìre ».

(26) Suet., Nero, 2 : per provinciam elephanto vectus est, lurba militum quasi inter solemnia triumphi prosequente. Nous ne pensons pas qu'il faille voir ici, comme on l'a fail parfois (E. PaIs, Fasti triumphales populi Romani, 2 éd., I, 1930, p. 48 et 367 ), l'indice d'un triomphe local de Domitius avant le triomphe romain, mais simplement l'indication de l'appareil guerrier par lequel le proconsul aimait à frapper l'esprit des populations.

(27) Voir les eléments de la discussion et la bibliographie dans JuLliax, III (1909), p. 19. n. 1. Depuis, E. Pass, o. l., p. 364 sq. Les textes sont rassemblés par .l. LebèGue, «Fasles de la Narbonnaise » dans l'Iistoire générale de Languedoc de dom Devic et dom Vaissete, 1. XV, 1892. p. 6-7. C. II. BExenict, The Iomans in Southern Gaul, American Journal of Philolog!, LXIII, 1942. pp. 38-50, en a repris l'examen critique. avec des observations intéressantes: il remarque notanment que seul Suétone (Véron, 2), par ailleurs si peu sùr, signale la présence de Domitius en Gaule pendant son consulat, en 122, et qu'il a pu arriver seulement en mars 121, suivi de peu par Fabius.

(28) Domitius a reçu le titre lors des victoires de 121 , dont la derniere est du mois daoût. L'usage du titre avant le triomphe (v. plus haut, n. 16 et 17 ) est attesté notamment par les inscriptions suivantes : C.I.L., I, $21614=$ II 5041 (en 189); 622 = III 14203, n०22 (en 16 $) ; 769$ el 
année doivent être exclus a priori pour la pose du milliaire : car un $\mathrm{XX}^{\mathrm{e}}$ milliaire fait partie d'une cuvre d'ensemble, le bornage de la Via Domitia, qui suppose accomplie ou très arancée la pacification du sud-ouest; elle n'a pu se faire en un jour. Mais il $y$ a plus : le lieu de la découverte indique que ces XX milles sont comptés à partir de Narbonne ${ }^{29}$. Il fallait donc que la colonie romaine fût fondée ou, à la limite, que son site fût officiellement décidé. Pour insérer le documenl dans son cadre historique, nous devons donc tenir compte des trois dates suivantes : I, la conquête de la vallée du Rhône; II, la fondation théorique, ou mieux : effective de Narbo Martius; III, le retour triomphal de Iomitius à Rome.

I. - La première est connue avec certitude: l'éclatante victoire de Fabius consul secondé par Domitius proconsul est du 8 août $121^{30}$.

II. - La seconde nous reporte beaucoup plus tard après cetle victoire que les quelques mois au minimum nécessaires à l'aménagement de la voie d'Espagne : c'est en 118 ou 117 en effet qu'à l'aide des lextes, on place la fondation de Narbo Martius, par une approximation dont il nous faut ici reviser les raisons. La fondation d'une colonie de citoyens romains est un acte important, qui s'accomplit en deux temps : le Sénat vote le principe de la fortdation sur un site déterminé; - la mission fondatrice se rend ensuite dans le pays et procède à l'installation des colons : c'est la "déduction " proprement dite. La colonie de Narbonne fut, après la Carthage de 123, qui n'a pas réussi, la première colonie de citoyens que Rome ait fondée outre-mer, et elle resta longtemps la seule ${ }^{31}$. Celle importante fondation nous est connue par

$2240=V I 37.045$ (en 89); I, 232828 (en 83); I. 21 720 = X 4751 (en 82); $746=11$, 531 (en 68 ). Ajouter I, $21630,692.741,768,746$, et les cas cilés par Momsen, Droit public, $l$. $l$, de l'emploi du titre par des $i$. qui n’ont jamais reçu les honneurs du triomphe. - Sur les monnaies: plus haut, n. 1ï; et: C.I.L., I, 22 3i9 (535), à Laodicée et .Ipâmée, en 56-53; 380 (526), ibid., en 53-51; 381 (52 7 ), à Laodicée, en 51; 377 (524), à Pergame, en 49-48; v. aussi 28i-289, 299, 336, 338, 354. et Grueber, o. $l$., II, p. 373 (en Espagne, en 39-3i): elc... Que M. Michel Labrousse, qui m'a aidé dans celte recherche, veuille bien trouver ici l'expression de mes remerciements.

(29) L'absence du nom de Narbonne sur le milliaire est normale: l'indication du point de départ ou du point d'arrivés est rare sur les milliaires de la République. mìme quand il y a deux ou trois chiffres de distances relatifs à des poinls différents. On ne connait que trois exemples, tous en Italie: C.I.L. I, 21 624 $(340)=8045$. (ienua, $C$ (remonam); I. $21661(561)=I X$. 5953, Roma; I, $2165 \tau$ (559) $=X 1,6.651$, risas. Le nom est d'ailleurs fréquemment omis sur les milliaires de l'Empire.

(30) Les textes datent la victoire du consulat de Fabius (121), et PLis: (II. N., VII, 50) précise le jour.

(31) Veld. Patenculus. II, i, 8 (éd. Stegmann de Prilzwad. coll. Teubner 1933): Prima autem extra Italiam colonia Carthago condila est; subinde Porcio Marcioque consulibus deducta colonia Narbo Martius. Toutefois il n'est pas impossible qu'il y ait eu des precédents (Carteia, en Espagne; aux Baléares, of. R.-E., IV, 516-517, J. Cakcopro, o. I., p. 25\%, n. 36). - Il n'y aura pas d'autres colonies en Gaule avant l'époque de César. 
quelques textes d'une grande concision qui ne distinguent pas entre les deux temps de l'opération. Sur les circonstances du vote, nous sommes renseignés par Cicéron : le projet se heurta à une certaine opposition avant d'être accepté grâce au talent du jeune C. Licinius Crassus, qui fut désigné pour diriger ia déduction ${ }^{32}$. Pour celle-ci, la date de 118 nous est fournie par les auteurs qui la placent sous le consulat de M. Porcius Cato et de Q. Marcius $\operatorname{Rex}{ }^{33}$, et M. Mattingly nous a appris, par une magistrale interprétation de monnaies frappées par Crassus et par son ami le jeune fils de Iomitius, que ce dernier a dirigé l'expédition avec Crassus ${ }^{34}$.

A partir de ces données, on a estimé différemment, par conjecture, le laps de temps qui a dû séparer le vote et la déduction. Les uns réduisent l'écart au minimum et placent, sans préciser, la "fondation" en 118 3.5. D'autres datent seulement la déduction de 118, estimant que la décision est antérieure à cette annće ${ }^{36}$. D'autres enfin placent, au contraire, la décision seule en 118, et, s"inspirant des usages relatifs à la nomination et à l'entrée en charge des magistrats, et de la date de 120 corrigée en 117 fournie par Saint Jéròme pour la déduction, abaissent à 117 le départ de la mission ${ }^{37} \ldots$ On ne peut faire un choix sans appel sntre ces différentes interpréta-

(32) Pro Cluentio, 51. 140 : bien des années après l'événement, Brutus plaidant contre Crassus fit remarquer que celui-ci, qui avait jadis rabaissé l'autorite du sínat «dans le discours par lequel il avail combattu une loi proposée contre l'établissement de la colonie de Narbonne » (in aissuasione rogationis ejus quae contra coloniam varbonensem ferebatur, quamlum potest de autoritate senatus detrahit), s'était contredit lui-mème par la suite «dans sa défense de la Loi Servilia en faisant de cet ordre un pompeux éloge et en se permettant contre les chevaliers romains les trails les plus mordants 》(Voir aussi De oratore, 2, 55, 223 et Quistilits, Inst. oral., $6,3,44)$. Ainsi, il y avait eu une proposition hostile à la fondation, done une proposition antérieure favorable. Mais cela ne veut pas dire que le Sénat ait réussi à faire repousser une fois cette première proposilion: Crassus a pu emporter la décision (Crc., Brulus, 43, 160: voluit adulescens in colonia . Narbonensi causae popularis aliquid arlingere eamque coloniam, ut fecit, ipse deducere) par le discours mème, à tendance démocratique, qui combattait la contre-proposition.

(33) Veld. Paterculus, 1 . l. et I, 15, 5: Narbo autem Martius in Gallia Porcio Marcioque consulibus ... deducta colonia est (il place ce consulat, correctement, en 118); - Ectrope, IV, 23 : M. Porcio Catone et Q. Marcio Rege coss... Narbonam in Gallia colonia derducta est: il place les consuls en 121, mais il faut décaler sa chronologie de trois ans (soit: 118), comme celle du chronicon de Saint-Jéròme qui indique: Narbonam coloniae deduclae à la $4^{\mathrm{e}}$ année de la $164^{\mathrm{e}}$ olympiade, soit en 120, à corriger en : 117, - sans qu'il y soit question des consuls.

(34) H. Mattixgly, some historical coins of the Roman Republic, Journ. of Rom. studies, XII, 1922, p. 233 (The foundation of Narbo Martius). Les monnaies sont antérieures à l'année 100; elles sont émises en deux séries, l'une pour la circulation a home, l'autre pour la Gaule, et sont frappées a l'effigie de la défaite gauloise. M. Carcoprso, o. l., p. 288, a entériné les conclusions de M. Mattrigay : voir sa belle page sur la fondation de Narbonne dans Points de cue sur l'impérialisme romain, p. 224-226.

(35) Opinion courante, adoptée par ae nombreux historiens, entre autres .I. Girkiskr, Vanucl, V, 1, p. 287.

(36) Jullax, III, p. 37: "Dès le temps de Domitius, je crois, il fut décidé que Narbonne serait la capilale de la Gaule transalpine, el qu'une colonie de citogens romains y serait envoyée»; p. 128, n. 7 : «la colonie, de citoyens romains. fut décidée sans doule avant cette date (118); le Sénat s'y opposa par une rogatio que combattit le fameux orateur L. Licinius Crassus dans un discours célèbre ».

(37) J. Cancopino, o. l., p. 287 : «La colonie de Narbonne (118)», et p. 288: «En 117, la 
tions de textes trop peu explicites, mais il est hors de doule que le consulat de Porcius et Marcius est lié indissolublement à la fondation de Narbonne, et que cette année 118 a réglé le sort de la colonie, soit "sur le papier", soit sur le terrain; elle l'a peul-êl'e même réglé des deux façons, car c'est la deductio que le textr le plus ancien place en 118 , et un précédent récent permet d'attribuer à une même année la décision et son exécution : c'est le cas de Carthage, dont la déduction a été commencée par Flaccus l'année même où elle a été décidée, en 12338.

La seconde condition nécessaire à la pose du milliaire est donc, en toute rigueur, celle-ci : que Domitius, vainqueur en 121, ait été encore présent en Gaule en 118. Il serait facile d'imaginer que Domitius ait borné prématurement la voie au sud de Narbonne, à partir du vieux site gaulois qui allait recevoir bientôt la forme d'une colonie romaine : l'existence de Narbo, ville indigène, est attestée par les auteurs plusieurs siècles avant la conquête romaine ${ }^{30}$, et l'on ne saurait douter que Domitius n'ait lui-même jeté son dévolı sur cet établissement, le plus important de la région, et ne l'ait désignć aux partisans de l'envoi d'une colonie ${ }^{40}$. Mais le bornage d'une voie est un acte officiel, et nous refuserons d'admettre, jusqu'à preuve du contraire, que la place gauloise ait servi de point de départ à ce bornage romain. Ce n'est pas parce que le bornage est antéricur a la fondation de Narbo Martius que le nom de la ville ne figure pas sur le milliaire : c'est parce que les noms de villes, nous l'avons dit, sont très fréquemment absents de ces monuments, surtout à cette époque. Le "cas-limite ", à nos yeux, c'est que Domitius ait borné la voie aussitôt acquis le vote sur la fondation, et le cas le plus vraisemblable, c'est qu'il l'ait fait aussitôt la colonie fondée. Il serait vain d'envisager l'hypothèse plus facile encore qui prêterait au fils la pose des milliaires marqués au nom du père: un milliaire n'est pas avant tout un monument commémoratif, sa pose est un acte administratif; attribuer le bornage au

colonie de Narbo Martius était inslallée par leurs soins »: p. 288, n. 60: fait stat des textes indiquant 118 , el du Chronicon de saint Jéròme (117), ef. plus haut, n. 33. M. MatTixgls; $o$. $l$., ne parle que de $11 \%$, sans explicalion.

(38) J. Carcopino, o. I., p. 260.

(39) Nous manquons d'une monographie hislorique sur la colonie. Outre l'llstoire générale de Languedoe déjà citée (réunion des textes), voir E. IEssarnoss, Géographie de la Gaule, I 1876. p. 248 s(1., II 1878 , p. 288; - JUllias, I 1907, p. 266, 400; IlI 1909, p. 37; V 1920, p. 36, n. 1; VI 1920, p. 348-353; - A. (iksilen, Mamuel, II. 2, 1934, p. 672-673 (oppidum de Montlaurès), p. 483)-492 (port de N., avec carte fig. 159), I 1931, p. 28i-288 (la colonie); - dom Lecherco, Narbonne du Diet. d'arch. chrét., I. XII, 1, 1935. col. 791-878; Pl. HÉLÉxa, Les Origines de Narbonne, Toulouse. 1937; - en dernier lieu : art. Varbo dans R.-k., Supplt., ‘. 1940, col. 515548, par P. Goessuer. Il est probable que l'emplacement de la colonie (l'actuelle Narbonne, aujourd'hui a 20 kilomètres de la mer) étàl, a l'époque préromaine, celui du port, de l'emporium de l'oppidrum de Montlaurès, silué à 4 kilomètres a l'ouest, où l'on a retrouvé des vestiges d'occupation depuis le VI siècle (Grkier, l. l.). Le peuple des Narbaioi serail cité déja par Hécatée de Milet, au Ve siècle av. J.-C., d'après Etienne de Byzance. El/hn., au mot Varbôn.

(40) V. plus haut, n. 36, l'opinion de Jullian. Lesistgut, $o, l$. 
jeune Domitius serait lui prêter, sans qu'on puisse faire état du moindre exemple, un faux dont nous n'avons pas le droit - si pieux fût-il - de charger arbitrairement sa mémoire. Bornage prématuré par le père, bornage après-coup par le fils, ícartons l'un et l'autre de ces "pis-aller ". Mais la date de 118 s'accorde-t-elle avec la troisième condition posée par le document lui-même : il est antérieur au retour triomphal à Rome de Domitius imperator?

III. - Sur cette troisième date, les historiens n'ont jamais été d'accord ì quelques années près, car on en était réduit pour la fixer à des conjectures tirées de textes indigents, la liste des "Fastes triomphaux" ne comportant plus la date des triomphes de Fabius ni de Domitius. Les éditeurs des Fasti ayant pourtant, depuis Sigonius, à la Renaissance, adopté par conjecture la date de 120 pour les deux triomphes, il nous faut reprendre la discussion, en considérant tout d'abord le texte de la liste triomphale ${ }^{41}$ :

Q. Fabiu. Q. Emiliani I. Q.n. an. $Đ C$...

Maximus procos. de Allobro(gibus)

't rege Arvernorum Betulto, $X \mathrm{~K} . \ldots$

Cn. Domitius Cn. f. Cn. n. Ahenobarb. a(n.) .. procos. de Galleis Arverneis, XVI K. ...

L'analyse de cette inscription, permet de fixer les points suivants :

$1^{\circ}$ J.es triomphes viemment (sans qu'il y ait de lacune dans la liste pour ces années) après le triomphe de Q. Caccilius Metellus Baliaricus (seul pour l'année 121) et avant les triomphes de 117 (il y en a deux, dont le second est de décembre). Ils ne peuvent se placer en 121, car Fabius est cité comme proconsul, et en 121 il était consul. Par contre, ils peuvent, a priori, se placer en 120, 119, 118 car Fabius et Domitius sont cités comme proconsuls et les Fasti ne mentionnent jamais l'itération des promagistratures. Ils peuvent même dater de 117, car les Fasti font état, par ailleurs, de plus de deux triomphes par an (quatre en 266, 241 et 175, trois en 167). Enfin quelles que soient la date ou les dates adoptées, il restera au moins entre 121 et 117 une année sans triomphe.

$2^{\circ}$ Fabius triomphe en premier. Ceci n'a jamais été contesté et résulte du fait que les Fasti respectent l'ordre chronologique sans exception connue, tout au moins dans l'ćtat fragmentaire où ils nous sont parvenus. Il s'ensuit que les deux triomphes n'ont pas eu lieu à moins de trois semaines de distance, au minimum: les jours $\mathrm{X}$ et XVI avant les Calendes ne peuvent en effet, dans cet ordre, s'appliquer qu'au 21 ou 22 d'un mois et au 15 ou 16 d'un mois postérieur. De plus, les Fasti distinguent bien les adverzaires de Fabius et de Domitius: ils ne font donc pas état d'une victoire

(41) Pour les Fasti, voir: C.I.L. I, 1 (2 éd.) 1893 (Mommsex); E. PAIS, Fasti triumphales populi Romani, I $1920=$ Fasti trionfali del popolo Romano I 1930; en dernier lieu A. DegrassI, Fasti triumphales dans les Inscriptiones Italiae, XIII, 1, 1947 ( 2 vol.) sans commentaire pour les triomphes en question, datés de 120: v. plus bas, n. 45. 
commune impliquant (ce n'est, d'ailleurs, pas une règle) des triomphes presque simultanés. Les triomphes peuvent donc avoir eu lieu soit dans une même année, soit en des années différentes.

$3^{\circ}$ Fabius triomphe comme proconsul. et Domitius, qui ne triomphe qu'après lui, ćgalement comm proconsul : Domitius a donc été prolongé en Gaule au moins aussi longtemps que lui. Fn 120, Fabius (consul en 121) est proconsul I, et Domitius (consul en 122) proconsul II. Il peut s'agir tout aussi bien de prorogations postérieures à 120, le nombre des prorogations n'élant jamais indiqué dans les Fasti.

$4^{\circ}$ Fnfin, Fabius triomphe sur les Allobroges it sur le roi des Aremes, Domitius sur "les Gaulois Arvernes", sans leur roi: il y a là, a priori, quelque chose d'insolite. Nous savons par ailleurs que les Allobroges ont été annexés, et que Fabius sera, devant la postérité, l' "Allobrogique "; les Arvernes, eux, sont restés libres... 42

L'inscription des Fasti ne permet donc pas, par elle-même, de choisir entre les années 120,119,118,117, ni d'affirmer que les deux triomphes aient eu lieu la même année. - Que nous apprennent les textes littéraires ? Florus, qui traite d'un seul triomphe et encadre sa relation des événements gaulois entre celle de la guerre de Jugurtha et celle de la guerre des Cimbres, ne fournit aucune précision d'ordre chronologique ${ }^{43}$. Eutrope, qui écrit près d'un demi-millénaire après ces événements auxquels il consacre trois phrases bourrées d'erreurs grossières ${ }^{44}$, donne deux indications: $1^{\circ}$ magna gloria consules ambo triumphaverunt, d'où E. Pais a conclu que les deux triomphes ont eu lieu coup sur coup; $-2^{\circ}$ il relate ces triomphes avant la fondation de Narbonne. Mais doit-on prendre au pied de la lettre ce résumé hâtif d'un auteur si peu sûr? D'ailleurs, consules ambo triumphaverunt peut fort bien signifier que les vainqueurs (il se trompe sur leurs noms) ont reçu tous deux les honneurs du triomphe; quarit à l'ordre dans lequel les triomphes et la fondation de Narbonne sont ici relatés, je ne sache pas qu'on en ait jamais fait état, ni ne crois qu'on puisse séricusement en tirer argument, pour placer le

(42) Pour la carrière de Fabius, v. plus bas, n. 56. Sur les Arvernes, César, B. G., I, 45.

(43) Flonus, I, 37, 5 : nihil lam conspicuum in triumpho quam rex ipse Bituitus. Il est le seul, avec Eutrope (v. n. suivante) à menlionner le triomphe.

(44) IV, 22-23 : Anno DCXXYII ab Vibe condila $627=127:$ non, l'intervention en Gaule est de 125) C. Cassius Longinus el Sex. Domitius consules (non: C. Cassius Longinus a été, consul, en 124, avec C. Sextius Calvinus) Gallis Transalpinis bellum intulerunt (non: c'est Fulvius Flaccus, consul en 125, qui a porté la guerre en Gaule; s'il s'agit de Domitius, c'est en 122 , suivi par Fabius en 121) et Arrernorum tune nobilissimue cicitati, atque eorum regi Bituito, infinitamque multitudinem juxla khodanum flurium interfecerunt: praeda ex torquibus Gallorum ingens Romon perlatu est. Biluitus se Domitio dedidil atque ab eo Romam ductus est, magnaque gloria consules (il s'agit. pour lui, des deux consuls précilés !) ambo triumpharerunt. M. Porcio Catone et (1. Warcio Rege coss., DCXXXIII anno ab Vibe Condila $(633=121$ : ces consulats sont de 118) Narbonam in Gallia colonia deducta est. Post a L. Metello et Q. Mucio Scaerola consulibus (non: ce Metellus a éle consul en 119, el Scaevola en 117) de Dalmatia triumphalum est (non: seul Metellus a triomphé, en 117 , el comme proconsul). On voit que, mème tenu compte du décalage de trois années qui permet de corriger les dates données par Eutrope, les erreurs ne manquent pas, ni sur les dates, ni sur les noms. 
retour de Domitius à Rome avant la déduction de la colonie ni, a fortiori, avant le vote qui l'a décidée. Par contre, un passage de Valère Maxime relatif aux agissements de Domitius en Gaule après la victoire d'août 121 a pu fournir a certains historiens et aux éditeurs des Fasti un argument favorable au choix de 120 pour le retour des vainqueurs, à d'autres une raison de prolonger le séjour de Domitius en Gaule au moins jusqn'en 119.

Au chapitre De la Perfidie, Valère Vraxime met sur le compte de la soif de gloire qu'il prête à Domitius l'action suivante: iratus namque Bituito regi Arrernorum quod [tum] suam et Allobrogum gentem, se etiam tum in provincia morante, ad Q. Fabii, successoris sui, dexteram confugere hortatus esset, per conloquii simulationcm arcessitum hospitioque exceptum vin.rit, ac Roman nace deportandum curacit $(I X, 6,3)$ : "Irrité contre le roi des Arvernes Bituit qui avait conseillé à son peuple et aux Allobroges, - alors que Domitius, prolongeait son séjour dans la province (ou : continuait d'exercer son commandement) encore à ce moment-là, - de se remettre à la discrétion de son successeur, $Q$. Fabius, il l'attira sous le prétexte d'un colloque et, l'ayant reşu comme hòte, il le retint prisonnier et le fit déporter par mer à Rome ". Et Valère Maxime ajoute que le Sénat, placé devant le fait accompli, se trouva fort embarrassé de la personne du roi et ne décida de le retenir en Italie que par crainte de voir les Arvernes se soulever à son retour ${ }^{46}$. D'autres auteurs donnent de la venue de Bituit à Rome une version différente: d'après l'abrévialeur de Tite-Live, il y serait venu de son plein gré par déférence pour le Sénat (on retrouve ici la même indication d'une démarche de Bituit prenant les devants, comme l'a vu Jullian, pour s'assurer l'initiative des négociations 47 : il n'avait été que l'allié des Allobroges, on ne s'était pas battu sur son territoire, et son peuple devait garder sa liberté). D'après Eutrope, - mais comment lui faire confiance ? - c'est Domitius lui-même qui, ayant reçu sa soumission, l'aurait amené à Rome ${ }^{48}$. Le texte de Valère Maxime est le plus détaillé des trois : voyons tout d'abord comment on l'a interprété en faveur d'un retour de Fabius au lendemain de la victoire, et d'un triomphe des deux vainqueurs ẹn 120 .

Pour les uns, il semble que la phrase "alors que Domitius prolongeait encore son séjour à ce moment-là " impliquerait, par opposition, que Fabius, lui, était reparti pour l'Italic: "la guerre terminée, écrit Jullian, le consul Fabius était retourné à Rome pour triompher de Bituit. Domitiusresta en Gaule pour organiser la nouvelle province (121) (se etiam tum im provincia morante) "49. On a dû conclure aussi de

(45) $0 . l ., 2^{e}$ éd., p. 368 . M. A. Degrassi, o. $l$, retient l'indication pour tout commenlaire sur la date de 120 qu'il adopte.

(46) Cujus factum senatus neque probare potuit neque rescindere voluit, ne remissus in putriam Bituitus bellum renoraret. Igitur eum Albam custodiae causa relinquit.

(47) Liv., Per., 61 : ipse cum ad satisfaciendum senatui Romam profectus esset, Albam custodiendus datus est, quia contra pacem videbatur, ut in Galliam mitteretur. - Jtulsas, III, p. 25: «l'Arverne sollicita ou accepta une entrevue avec ses vainqueurs, afin de diriger lui-mème les négociations ».

(48) V. plus haut, n. 44. Nous arons dit le peu de créance que l'on doit faire à cel auteur, notamment pour ce passage.

(49) III, p. 35 , et n. 3 ; p. 25 , n. 3 : «selon Valère Maxime, Bituit se serait adressé à Fabius, 
l'ensemble du passage que Domitius n'a pu procéder à la capture de Bituit qu'après le départ de Fabius 50 . L'interprétation est done la suivante: l'initiative de Bituit et sa capture ont dô suivre d'assez près la défaite d'août 121; comme elles se placent après le retour de Fabius et avant son triomphe où le roi a figuré, il s'ensuit que Fabius est rentré peu après la victoire de 121. On a prêté, en conséquence, à Fabius une certaine hâte de revenir à Rome cueillir les lauriers du triomphe, à Domitius une rertaine rapidité dans la pacification du sud-ouest gaulois et dans l'organisation de la conquête, et on les a fait rentrer et lriompher tous les deux, à quelques mois de distance, au cours de l'année 120 ¿1.

D'aulres cependant ont compris le texte différemment. "Comme Fabius a triomphé arce le titre de proconsul, a écrit Albert Lebègue, il est probable que la Province lui fut attribuée l'année suivante (20), et cependant Domitius y est demeuré. Nous savons, en effet, par Valère Maxime, que Bituit, voulant se livrer à Fabius, "successeur de Domitius", ful attiré par ce dernier et fait prisonnier. Domitius l'encoya par mer à Rome, sans doute parce que la Province était sous l'imperium de son collègue " i2: tout s'est donc passé, pour Lebègue, alors que Fabius était en Gaule. Pour mesurer la valeur de ces opinions divergentes, reprenons le texto de plus près.

Valère Maxime relate deux faits, qui découlent l'un de l'autre, mais il ne les présente nullement comme immédiatement consécutifs: $1^{\circ}$ l'initiative de Bituit provo(quant la colère de Domitius (quod... horlatus essel); $2^{\circ}$ la vengeance de Domitius (vinxil). - deux actes d'un petit drame dont l'épilogue sera la présence du roi prisonnier au triomphe de Fabius. Or, c'est de toute évidence au premier acte, et à lui seul, que se rapporte se etiam tum in provincia morante, placé en incidente dans la

¿ la grande colère de Iomitius. Fabius filant reparti po:ur l'Italie, c'est Domitius qui devait en effet diriger les négociations ».

(50) Le texte n'a jamais 'sté discuté de très près : mais cette interprétation justifie seule la façon dont on présente les faits : par ex. E. Dessardiss, Géographie de la Gaule, II, p. 281 : "Fabius retourna à Rome, laissant en Gaule Cn. Domitius pour pacilier le pays; mais cclui-ci, froissé de ce que Bituitus avait conseillé à ses alliés de se soumetlre à Fabius et non à lui, s'empare traitreusement de sa personne et l'envoie à Rome».

(51) E. DFsJaliotss (o. l., p. 287) a fait rentrer Fabius dès 121 «avant l'expiration de son annéc de consulal et mème avant la fin de la campagne», ce qui esl impossible puisqu'il a triomphé comme proconsul. - Iprès la victoire de 121, Fabius «se dirigea vers l'Italie où il avait hâte de célébrer son (riomphe »; Domilius « rentrait à Rome, quclques mois après Fabius et triomphait à son tour »: tous les deux en 120 (J. Garcopro, o. l., p. 285-286). - «Domitius resta en Gaule pour organiser la nouvelle province (121), et il s'acquitla de sa lache en vrai Romain, voyant très vile la chose a faire, et la faisant aussitot sans autre souci que l'intérèt de l'Empire» (Julıın, III, p. 35); - «Il est possible que, Domitius ayant été laissé en Gaule (cf. Valère-Naxime, IX, 6, 3), ce fut pour organiser cette province, et que cetle organisalion, par suite, se place en 121 ou 120. On place son triomphe et celui de Fabius à Rome en 120, mais sans aucune certitude » (ibirl., p. 20, n. 2); - «Mais la rapidité des triomphes de Fabius ct de Domilius doit ètre une raison de plus pour admirer leur cuvre, achevée en un an, par deux batailles, avec une perte presque nulle de sang romain; - le plus puissant des empires occidentaux détruil pour toujours; la conlrée disloquée en deux tronçons, qu'un millénaire ne parviendra pas à rapprocher, etc... » (ibid., p. 31). On voit que Jullian, tout en affirmant le départ de Fabius après la victoire de 121 , est réservé sur la date des triomphes.

(52) Fastes de la Narbonnaise, o. l., p. 9. La prolongation de Domitius jusqu'en 118 a été admise nolamment par Fischer et par Zumpt, v. plus loin, n. 59; et la prorogation jusqu'en 119 seulement, d'après Lebègue, par W. F. JASHEMSkI, The origine and history of the proconsular and propraetorian imperium to 27 B. C., Chicago, 1950, p. 65, n. 1 et 2; et p. 140. 
phrase qui expose seulement l'initiative de Bituit (quod... horlatus esset): el tout ce que nous apprend cette phrase, c'est que Iomitius était encore là, prolongeant son séjour, quand Bituit a choisi de s'adresser à Fabius. Il ne saurait done être question d'induire de se... morante que Fabius, contrairement a Domitius, ne prolongeail pals son séjour, done était déjà reparti à ce moment-là; au contraire, comment admettre, en effet, que le roi des Arvernes ait eu l'idée de s'adresser à Fabius, comme au successeur de Domitius, s'il avait déjà quitté la Gaule? On ne peut admettre non plus, puisque Fabius est resté comme proconsul en Gaule au moins une partic de l'année 120 (nous le sarons par les Fasti), que Bituit et les Allobroges aient attendu plus d'un an après leur défaite d'août 121 pour faire leur soumission, ...une fois Fabius reparti. Il faut done bien comprendre, avec Lebègue, que le roi s'est adressé à Fabius alors en Gaule, en tant que "sucesseur de Domitius, comme si ce dernier itail reparti, "alors que (au contraire) il prolongéait encore son séjour dans la prorince à re moment-là": d'où sa colère. Le mot "successeur" a ici son importanen: est-ce seulement de la succession de Domitius au consulat qu'il s'agit? Celte succession n'a de sens, aux ycux du roi des Arvernes, qu'en tant qu'elle se double de la succession aux affaires de Gaule, et il est remarquable que, Fabius étant considéré peu après sa victoire comme le successeur de Domitius en Gaule, ce dernier soit néammoins resté comme proconsul en même temps que lui en 120.

Pour le reste, on en est réduit aux conjectures. Fabius a-t-il effectivement reçu la soummission des Allobroges et de leur allić ? On doit le croire, car il triomphera sur les uns et sur l'autre, et devant l'Histoire il sear l' "Allobrogique": il faul, alors, qu'il ait laissé sa liberté à Bituit, puisque c'est Domitius qui l'a réduit, par traîtrise, en caplivité; cela n'a d'ailleurs rien d'impossible, car nous savons que le Sénat ne s'attendait nullement à la capture du roi, el que les Arvernes sont restés libres : Fabius n'avait done point d'instructions contraires à la liberté de Bituit. D'autre part, Domitius a dû agir aussitôt après le départ de Fabius, car le roi captif est arrivé à temps pour figurer au triomphe, qui, selon l'usage, a dû suivre d'assez près le retour de Fabius. C'est le plus vraisemblable... Mais on voit qu'une fois bien distingués les deux temps de cette histoire, si l'on peut situer avec viaisemblance le premier dans les mois qui ont suivi la victoire, rien ne permet d'en faire autant pour le second, ni d'y voir une indication ferme concernant le départ de Fabius et, encore moins, la date de ce départ.

En bref, le texte de Valère Maxime a pour mérite essentiel d'être pour nous l'écho d'une pique d'amour-propre entre Fabius et Domitius, et peut-être d'une rivalité plus profonde en confirmant la prolongation de Domitius qu'attestent les Fasti, aux còtés de Fabius. Mais sur la date de leurs retours, il ne nous apprend rien, et sur l'ordre même de ces retours, nous n'avons comme témoignage que l'ordre de leurs triomphes sur la liste capitoline.

Après cel examen des texles, nous ne saurions donc mieux faire que de reprendre à notre compte la prudente réserve dont C. Jullian, dans une note, a tempéré sa conjecture relative au retour de Fabius: "On place le triomphe de Domitius et de Fabius à Rome en 120, mais sans aucune certitude " 53 .

(53) III, p. 20, n. 2. V. plus haut. n. 51. 
L'inscription des Fasti el les textes laissent bien le choix, pour ces triomphes, entre les annćes 120, 119, 118 et même 117.

Mais il nous reste à examiner une dernière paison que l'on a invoquée contre la prolongation de Domilius jusqu'en 118. On a fait valoir le fait que Narbonne a été fondée par d'autres que Iomitius. Cetle fondation n'ayant eu lieu qu'en 118, on n'a vu aucune nécessité à prolonger le séjour du proconsul jusqu'à cette date, et même on a hésité à le faire rester jusque là pour repartir précisément au moment où arrivaient les fondateurs de la colonie. Mais on peut répondre avec Lebègue à cette objection : "Domitius ", dit-on, "n'a pas fondé Narbonne. Ce n'est pas une preuve. Un consul, un proconsul, pouvaient établir dans la province qu'ils gouvernaient un camp fortifié, une garnison permanente. Mais à cette époque les colonies de citoyens romains partaient d'Italie, étaient créées par le Sínat et conduites par les triumvirs, en vertu d'une délégation extraordinaire et spéciale. Ces triumvirs et les gouverneurs des provinces où s'établissaient les colonies avaient donc des pouvoirs et des attributions distinctes $"{ }^{54}$. De plus, et surtout, nous savons aujourd'hui, grâce à M. Mattingly, que la fondation de Narbonne a été, elle aussi, cuvre " domitienne ", par la nomination du fils du proconsul comme curator coloniae deducendae aux côtís de son ami Crassus. La non-participation de Domitius à cette fondation cesse du même coup de plaider en faveur de son départ dès l'année 120: bien au contraire, voilà rendue parfaitement logique sa présence en Gaule jusqu'aı moment où, laissant à son fils le soin de couronner son œuvre qu'il peut considérer comme virtuellement achevée, il renonce à demander une prolongation devenue de plus en plus difficile à obtenir, et part l'esprit tranquille. Il est non moins normal, dans ces conditions, qu'il n'ait pas assuré lui-même les destinées de la colonie : une telle fondation est œuvre de longue haleine, à laquelle plusieurs années suffisent a peine; Domitius, quittant la Gaule en 118 ou en 117, passait la main et le flambeau à quelqu'un qui, cette fois, était un "successeur " de son choix.

Ainsi, ni nos sources, ni le contexte historique n'imposent pour le triomphe de Domitius une date antérieure à l'année 118, alors que la découverte du milliaire constitue à son endroit la plus formelle des contre-indications : les trois conditions posées par le document sont bien compatibles entre elles.

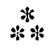

Il nous reste, avant d'adopter cette date de 118 ou de 117 pour la pose du milliaire, à mesurer et à vérifier les conséquences qu'elle entraîne, en

(54) O. l., p. 11 . 
retraçant les faits tels qu'ils découlent essentiellement de la plus sûre de nos sources, la liste des Fasti triumphales.

a) Fabius et Domitius ont été proconsuls ensemble en 120, au moins, et peut-être plus longtemps: cette présence simultance de deux proconsuls au delà des Alpes demande explication. Théoriquement, en effet, Fabius, successeur de Domitius au consulat, n'eûl-il pas dû lui succéder aux affaires de Gaule ? Si la victoire décisive n'avait pas été acquise en août 121, c'est l'un des consuls de l'année 120 que l'on en̂t envoyé en Transalpine, où Fabius restait normalement comme proconsul. Si Domitius y est demeuré, c'est qu'il a obtenu de rester en plus de Fabius, ce qui constitue, on ne l'a pas assez souligné, un succès certain à son actif, moins sur le Sénat que sur Fabius. Tout s'est passé comme s'il y avait eu épreuve de force entre Fabius et Domitius: une fois franchi ce cap, d'autres prorogations ne devaient plus soulever de difficullés. Le texte de Valère-Maxime tel que nous l'arons interprété commenterait précisément l'indication des Fasti.

Cette coexistence de deux proconsuls ou personnages proconsulaires dans une même région n'est pas un fait isolé: on en a des exemples présent, elle s'explique suffisamment par le fait que la Gaule n'était nullement en 120 une province bien délimitée, mais une conquête toute récente, encore fragile, qui demandait à être ítendue jusque vers les Pyrénées. Et l'on envisage très bien le partage du commandement que suppose la coexistence des proconsuls: Fabius au nord, au contact de la route d'Italie par les Alpes, près de ces Allobroges auxquels il devra son surnom; Domitius au sud-ouest, dans ces contrées dont l'artère vitale lui devra son nom. L'un et l'aulre noueront pour leur descendance des liens durables avec l'un et l'autre pays ${ }^{56}$.

(55) Voir les listes, très uliles, des magistrats, promagistrats, ou mirali à pouvoir proconsulaire de la République, classés par provinces dans V. F. JAshisski, o. l., 1950, p. 100-160. Pendant la première guerre punique, on a vu M. Emilius Paullus et Ser. Fulvius Paetinus Nobilior, consuls en 255, défaire la flotte carthaginoise all cap d'Hermès el célébrer leurs triomphes de Cossurensibus et Poeneis en 254. - En Espagne, avant que le pars conquis fùt scindé en deux provinces (197), on a vu, de 215 a 198 , le pouroir proconsulaire conféré chaque annés à deux pricati, dont certains furent longuement prorogés. - En Macédoine, en 118, L. Caecilius Melellus proconsul a eu auprès de lui Cn. Cornelius Sisenna, prèteur à imperium proconsulaire: en T2, (C. Seribonius Curio et M. Terentius Varro Lucullus y sont tous deux proconsuls ainsi qu'en 64 Q. Caecilius Melellus Creticus et L. Manlius Torqualus. - En Cilicie, sont proconsuls ensemble: en 74 , P. Servilius Vatia Isauricus et L. Octavius. - en $6 \tau$ L. Licinius Lucullus et Q. Marcius Rex. - en 53 P. Cornelius Lentulus Spinther et $\Lambda$ p. Claudius Pulcher, en 51 ce dernier et M. Tullius Cicero. - En Syrie, en 38, P. Ventidius et C. Sosius. - Nous ne comptons pas ici, sauf pour l'Espagne, les nombreux exemples de deux pricati, prèteurs ou proprêteurs ạaant dans une même province l'imperium proconsulaire. W. F. Jashemski met bien en lumiere le fait que les consulaires sont envoyés particulièrement dans les provinces présentant un intérèt militaire.

(56) Pour les Domitii, Julliax, III, p. 38, n. 1; 63, n. 4; 282, n. 3, 581, n. 1. Pour les Fabii, R.-E.. VI (1909), n 110, coll. 1796 (Münzer), et Cic., Pro Fonteio, 15: apud illos (les Allobroges) Fabiorum nomen est amplissimum. 
b) Fabius a triomphé sur les Allobroges et sur le roi des Arvernes, Domitius n'a triomphé que sur "les Gaulois Arvernes ", sans leur roi: quelles que soient la date et les modalités de la prise de Bituit, on est en droit de penser que Fabius l'a fait figurer à son triomphe par un coup en relour que Domitius présent à Rome eût certainement prévenu, car il s'efforcera plus tard nous le savons - ainsi que ses descendants, d'en effacer la trace en " confisquant à son profit ", comme l'a très bien vu Jullian, le souvenir de la victoire ${ }^{57}$. On sait la suite: Fabius cuntinuera d'avoir les plus grands honneurs, il sera le premier Romain à élever sur le forum un arc de triomphe; Domitius n'aura pas cette distinction éclatante, mais il aura la gloire sans doute plus durable d'avoir donné à Rome la voie d'Espagne en organisant l'ensemble de la conquête.

c) Fabius a triomphé le premier: il a donc dû rentrer avant Domitius, mais on ne saurait évaluer la durée de son séjour, tant l'œuvre qu'il a dû accomplir en Gaule, après la victoire de 121 nous est mal connue. Elle cst certainement moins importante que celle de Domitius. Rien n'interdit pourtant de prolonger son sćjour concurremment avec celui de Iomitius ${ }^{58}$, car nous savons peu de chose de sa carrière officielle après son retour; mais rien ue permet non plus d'affirmer cette prolongation. Par conséquent, si l'on place le triomphe de Domitius en 118 ou en 117, Fabius peut avoir triomphé soit trois ans (120) ou deux ans (119) ou un an (118) avant son rival, soit la même année que lui, mais au moins trois semaines avant lui.

d) Domitius a triomphé le second: l'ampleur de l'œuvre qu'il accomplit en Gaule après 121 a été souvent invoquée pour justifier sa prolongation en Gaule jusqu'en 119 ou même en 118 50. "Ecrasés par Fabius, a écrit par exemple Lebègue, les Allobroges ont fait leur soumission, et si les Romain.s ont retenu, contre toute justice, Bituit et son fils, cela prouve qu'ils avaient encore à craindre les Arvernes. Domitius serait donc resté en Gaule après le départ de Fabius... On a une autre raison de croire qu'il est resté dans le pays pour l'organiser: la voie Domitia porte son nom... Le soin de pacifier et d'organiser la Province put retenir Domitius assez longtemps en Gaule. On est donc fondé à croire qu'il y resta, non seulement pendant l'année où Fabius

(57) III, p. 26, II. 2, à propos de la série de monnales qu'a depuis, si bien expliquée M. Mattingly: "Sans doutes, les monnaies de la gens Domitia représentent un guerricr gaulois, debout sur son char, lançant le javelot; mais rien ne prouve, ou qu'il s'agisse de Bituit, ou que la gens Domitia n'ait pas essayé de confisquer à son profit le bénéfice de la victoire ».

(58) Pour les exemples de prolongation simultanée de deux privati a pouvoir proconsulaire ou de deux promagistrats, v. plus haut, n. 55.

(59) Entre autres, E. W. Fischen, Römische Zeittafeln, Altona, 1846, p. 153; Aug. ZuMPT, De Gallia Romanorum provincia, dans Studia Romana, Berlin, 1859, p. 118. 
fut proconsul, mais aussi l'année suivante, et qu'il fut proconsul en 119 av J.-C. " ${ }^{60}$. A l'appui de cette appréciation, il n'est pas inutile de rappeler ce, que fut cette œuvre personnelle de Domitius en Gaule. Après avoir érigé avec Fabius des trophćes destinés à commémorer la victoire de la vallćc da Rhône, il guerroie sans doute encore contre les Arvernes ${ }^{61}$, puis il descend cette vallée; refoule les Rutènes au nord des Cévennes ${ }^{62}$, mâte au passage les Cavares du Comtat, les Helviens du Vivarais ${ }^{63}$, puis se lance à l'ouest du fleuve, soumet le grand peuple des Volques Tectosages qui dominait jusqu'à Toulouse où il installe une garnison ${ }^{64}$, et tout particulièrement la tribu des Elisici dont Narbo était la capitale, qu'il désignera pour recevoir la future colonie et pour donner à Rome le premier port de l'Occident après Marseille. Il noue peut-être une alliance avec le roi de Lectoure, qui tenait l'arrièrepays de Toulouse avec la région du Gers ${ }^{6}$. Il étend alors son contrôle jusqu'aux Pyrénées et, au fur et à mesure de cette pacification dont nous connaissons seulement les grandes lignes, il aménage la voie d'Espagne et fonde sur son passage, prìs d'Agde, Forum Domitii ${ }^{66}$. Cette cuvre variee, aussi puissante qu'originale, où l'orgunisation va de pair avec la pacification. n'a pu, de toute évidence, s'accomplir en quelques semaines: Domitius a dù la construire troncon par tronçon, tout au long de la voie sans laquelle eile n'eût rien été. Et l'on a récemment supposé que, les peuples du Sud-Ouest étant, d'après Strabon (IV, 2,3), clients des Arvernes depuis les abords de Marseille jusqu'aux Pyrénées, c'est l'œuvre d'ensemble de Domitius qu'à consacrée son triomphe "sur les Gaulois Arvernes ", tandis que Fabius triomphait des Allobroges et de Bituit parce qu'il avait reçu leur soumission ${ }^{67}$.

Notre proposition sera donc la suivante: Domitius est resté en Gaule non seulement en 119 mais encore au moins une partie de l'annće 118, pour

(60) O. l., p. 9.

(61) Jullian le suppose, III, p. 19, n. 1. Cela achèverait d'expliquer, comme l'a vu Lebègue (plus haut, n. 60), son triomphe de Galleis Arrerneis.

(62) César, B. G., I, 45, 2, attribue le fait à Fabius: M. Carcopro admet, o. l., p. 286, n. 51, que c'est par liaine des Domitii. Sur le démembrement des Rutènes que Jullian, III, p. 22-23, plaçait a ce moment-la, cf. en dernier lieu $\Lambda$. Albexque, Les Rutènes, 1948, p. is, qui le place seulement au Ier siècle.

(63) Ceci résulte du fait que ces peuples se trouvaient sur son passage: cf. JLLLis, III, p. 22, n. 1 .

(64) La garnison que signale Dion Gassius, XXVII, fragm. 90 (éd. Boissevain, p. 334), au moment de la guerre des Cimbres, doit en eff el remonter à ce moment-là.

(65) J. Carcopino, o. l., p. 286, n. 54, fail justement remonter à cette époque l'alliance que Jullian. III, p. 28, n. 5, a placée vers 67.

(66) Le nom seul l'indique, mais avec la plus grande vraisemblance. Il apparâit dès l'épaque d'Auguste, sur les gobelets de Vicarello. Il fallait un personnage aussi important que Domitius pour donner son nom à un établissement. Parmi ceux de ses descendants qui furent mèlés aux affaires de Gaule, aucun ne semble avoir eu l'autorité nécessaire.

(67) C. H. BENEDict, o. l., p. 49. 
accueillir et installer son fils chargé de fonder la colonie sur le site de Narbonne qu'il avait choisi lui-même, et pour consacrer officiellement à la veille de son départ l'aménagement de la Vıa Domitia par un bornage dont le premier témoin vient de parvenir jusqu'à nous ${ }^{68}$. Il est même possible qu'il soit rentré seulement en 117, car pour cette année la liste des Fasti ne compte que deux triomphes: celui de Domitius, qui vient aussitôt avant, mais sans sa date, peut done fort bien appartenir aussi à cette année-là, car, nous l'avons vu, il y avait parfois trois et jusqu'à quatre triomphes par an. Mais 117 est bien la dernière limite, puisque le triomphe de Domitius est cité avant ceux pour lesquels cette date nous a été conservée.

Un texte bien connu de Polybe pose ici une délicate question. Décrivant la voie d'Espagne au Rhône, il écrit: " De Carthagène à l'Elbe, il y a environ 2.600 stades; de l'Elbe à Emporion, 1.600 (d'Emporion à ${ }^{* *}$, environ 600); ct de là au passage du Rhône environ 1.600 ", et "il ajoutc: "Cctte distance est maintenant mesurée et marquée de 8 en 8 stades, soigneusement, par les Romains; du passage du Rhône, etc... ". De 8 en 8 stades, c'est-à-dire à peu de chose près, de mille en mille. Polybe aurait-il connu la Via Domitia, bien qu'il ne parle pas de la conquête, de la Narbonnaise ? On ne peut malheureusement l'affirmer, car on ignore la date de sa mort, qui, d'après les maigres renseignements que nous possédons, eut lieu au plus tôt en 128/127, au plus tard en 118/117. Pour qu'il soit mort (à 82 ans comme nous l'apprend le Pseudo-Lucien) seulement en 118/117, et qu'il ait ainsi eu le temps d'introduire dans son texte le renseignement qui authentifiait les chiffres de distance donnés pour la voie d'Espagne, il faudrait qu'il ait eu à peine 18 ans lorsqu'en 181/180 il accompagna son père dans son ambassade en Egypte: et cela n'est pas impossible, car il nous avertit qu'il était alors au-dessous de l'àge légal, mais sans nous dire de combien. Le cas est cependant "limite ": et, dès avant la découverte du milliaire, alors qu'on manquait de certitude su! la création de la voie par Domitius, on avait douté de l'authenticité du texte ${ }^{68}$. De toute façon, ce passage se présente comme une addition (" cette distance est maintenant mesurée, etc. "), due à Polybe ou à un interpolateur, au texte du livre III qui a certainement été publié par Polybe bien avant la fin de sa vie. Si tentant que cela soit, on ne peut aujourd'hui affirmer que

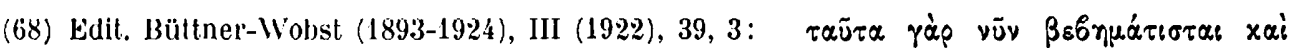

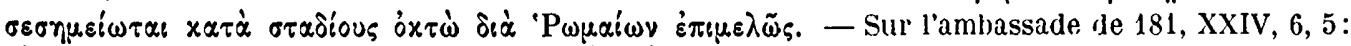

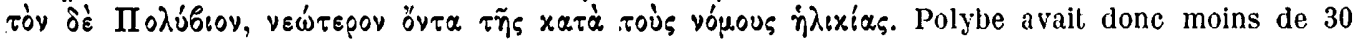
ans en 181-180 et il avait au moins trente ans en 169-168 lors de son hipparchat (XXVIII, 6, 9). D'apris le Pseudo-Lucien (Macrob., 22), il est mort entre 81 et 82 ans d'une chute de cheval. Que M. André $A$ ymard, qui a bien voulu me renseigner sur la biographie de Polybe, trouve ici l'expression de mes vifs remerciements. 
le texte est bien de Polybe et que celui-ci n'est mort qu'en 118/117. Si Domitius quitte la Gaule dans le deuxième semestre de 118 ou de 117, son séjour aura duré trois ou quatre années pleines après la victoire décisive d'août 121: la perspective ne parait point fausse, en vertu de laquelle on est conduit à les lui accorder.

Cette perspective qui, appliquée à l'œuvre accomplie en Gaule, nous paraît juste, le reste-t-elle si l'on se place sur le plan plus général de l'histoire romaine ? Ce quatrième, peut-être ce cinquième proconsulat dont la découverte du milliaire nous incite à gratifier. Domitius, sont-ils admissibles en ce début du dernier quart du $\mathrm{II}^{\mathrm{e}}$ siècle ? La raréfaction de la prorogation au $\mathrm{II}^{\mathrm{e}}$ siècle, dùe à la méfiance du Sénat à l'égard des généraux, est un fait reconnu ${ }^{69}$. Elle n'est point telle, toutefois, qu'on ne puisse attribuer à un Domitius un séjour total, au delà des Alpes, d'un peu plus de quatre ou de cinq années, en y comprenant une partie de son consulat ${ }^{70}$. En Corse et en Sardaigne, en effet, nous trouvons L. Aurelius Orestes, consul, en 126, et proconsul de 125 à 122, puisqu'il n'a triomphé qu'en 122; puis M. Caecilius Metellus, consul, en 115 , et proconsul de 114 à 111 , puisqu'il n'a triomphé qu'en $111^{71}$ : deux fois, presque de suite, une quadruple prorogation est donc accordée à un consulaire, précisément à l'époque qui nous intéresse, et dans une province déjà ancienne. Le fait n'est-il pas plus normal encore dans cette Gaule méri. dionale seulement à moitié conquise en 121, la seule région dont Rome pût alors redouter le voisinage immédiat, province " entourée de trop d'ennemis, trop récemment conquise, composće d'éléments trop disparates, trop éloignée de Rome et trop importante à conserver aux portes de l'Espagne " (Lebègue) pour qu'on pût impunément interrompre deux années durant son organisation ? Bientôt viendront par là les Cimbres, dont le passage provoquera des prolongations consulaires bien autrement considérables et lourdes de conséquences....

Dans ces conditions, auxquelles il ne faut pas craindre d'ajouter la condition psychologique - la personnalité exceptionnelle d'un Domitius, jalonnant de son nom et du souvenir anticipé de son triomphe la voie qui traverse sa conquête, forgeant de ses propres mains la nouvelle province de Rome dont il n'oublie pas de faire une sorte de fief pour sa descendance ${ }^{72}$ et qui sera

(69) V. J. Carcopino, o. l., p. 45.

(70) Sur la date de son arrivée en Gaule, d'après G. H. Benkdict, v. plus haut, n. 27.

(71) W. F. JASHEMSKI, o. l., p. 120, d’après J. KLEIN, Die Verwaltungsbeamten der Proviñen des röm. Reiches, I, 1, Sicilien und Sardinia, 1878, p. 199-249.

(72) V. plus haut, n. 56. Rappelons les faits les plus marquants: son fils Cneus, fondateur de Narbonne en 118, qui fait frapper monnaie au souvenir de la victoire paternelle, et dirige en 104 ou 103 l'accusation contre Silanus, defait par les Cimbres; son petit-fils Lucius, gourer- 
la gloire la plus sûre de sa race, - il n'y a pas lieu d'hésiter à prolonger jusqu'en 118 au moins et peut-être jusqu'en 117 ce proconsulat essentiel dans I'histoire de la Gaule: car cette date de 118 ou de 117 que le milliaire suggère pour le retour triomphal de Inomitius à Rome, ne se heurte, en dernière analyse, à aucune difficulté.

La découverte du milliaire de Domitius éclaire ainsi d'une même et nouvelle lumière la carrière de Domitius, l'œuvre accomplie par Rome outremonts, et l'empreinte civilisatrice reçue alors par la Gaule méridionale. Tout d'abord, elle supprime toute lacune dans la carrière du conquérant: consul en 122, Domitius reste proconsul jusqu'en 118 ou 117 inclus et laisse alors son œuvre aux mains de son fils; il triomphe à la fin de l'année et, après deux ou une années révolues, il accède, en 115, à la censure ${ }^{73}$. Il est ainsi parvenu par une ascension continue jusqu'au faîte des honneurs militaires et civils auxquels un Romain de son temps pouvait prétendre. Il a ajouté au pays allobroge, qui restait le don de Fabius à la patrie romaine - don auquel il n'aura pas été lui-même étranger -, la conquête de ce " Far West " gaulois où se sont affirmés dans loute leur force son tempérament hardi et son génie créateur. Il s'est inscrit ainsi sur la liste des grands pionniers qui auront eu la possibilité de modeler de leurs mains, à mesure qu'ils le pacifiaient, le pays auquel ils imprimaient la marque indélébile de la civilisation qu'ils représentaient. Son cuvre a le caractère et le cachet personnel de celle des bâtisseurs d'empires : si la Narbonnaise est, en apparence, à moitié " fabienne ", elle est en réalité plus qu'à moitié " domitienne ".

En second lieu, toute lacune est du même coup supprimée dans l'œuvr accomplie alors par Rome au-delà des Alpes: il n'y a plus de périlleuse interruption dans cette conquête d'une importance vitale; il n'y a plus, non plus, de hâte dans la mise en place des éléments si divers qui constitueront la première province arrachée aux Gaulois d'outre-monts. De 125, date où pour la première fois une armée consulaire passa les Alpes sous la conduite de Flaccus, à 115, date la plus haute que l'on puisse admettre pour le retour en Italie des fondateurs de Narbo Martius, Rome n'a cessé d'envoyer en Gaule ses meilleurs hommes de guerre, consuls ou consulaires, puis ses meilleurs hommes d'avenir. On l'a déjà exprimé fortement: " En Gaule, la politique n'avait été nullement influencée par l'acharnement des luttes qui sévissaient fondation de Narbonne, si l'on accepte l'interprétation de M. Mattingix, $\circ . l$.

(73) V. sa carrière, R.-E., V (1905), col. 1322-1324, s. v. Domitius, nº 20 (MüNzER). 
dans la Ville, et d'année en année, au contraire, elle avait, d'un mouvement ininterrompu, agrandi le champ de ses opérations et de ses victoires " ${ }^{74}$. Ce n'est pas le moindre intérêt de la découverte d'un milliaire de Domitius, que de délivrer décidément de toute solution de continuité celte fruclueuse période de la conquête romaine qui a vu naître " la Province " ${ }^{75}$, el s'élever des Alpes aux Pyrénées ce pont immense dont la pile maîtresse était Narbonne.

Enfin, la romanisation du Midi gaulois prend de ce fait une valeur nouvelle. Elle n'est plus la conséquence d'une victoire-éclair en 121, suivie d'une " promenade " de Domitius jusqu'aux Pyrénées, puis, après un abandon temporaire, d'une consolidation après-coup, à partir de 118. L'affaire de Gaule a été moins vite réglée qu'on ne l'a dit ${ }^{76}$; le maintien et l'organisation de la conquête ont demandé l'effort prolongé d'un Fabius et d'un Domitius; l'unité n'a été enfin scellée, par le fils de ce dernier, qu'avec la première pierre dé la colonie nouvelle. Cette romanisation, on ne comprendrait pas, en vérité, qu'elle ait pu être si profonde et si durable, si elle avait été à ses débuts épisodique, ou superficielle, ou aucunement contrariée.

\section{Paul-Marie Duval.}

(74) J. Carcopixo, o. l., p. 284.

(75) Sur les différentes dates proposées pour l' «ère» de la province, cf. Jullias, III, p. 20, n. 2 (Domitius, ou Marius), et son opinion: «il est possible que, Domitius ayant été laissé en Gaule (cf. Valère-MAXINE, IX, 6, 3), ce fut pour organiser cette province, et que cette organisation, par suite, se place en 121 ou $120 »$. La découverte du milliaire, première preuve concrète de l'œuvre administrative accomplie par le conquérant, vient à l'appui de cette opinion, mais nous porte à adopter plutôt l'année 118 ou l'année 117.

(76) G'est cette impression de facilité dans la conquète qu'a donnée beaucoup plus tard, pour des raisons trop claires, AMMEN ManceldiN, XV, 12: IIae regiones, praecipue quae confines Italicis, paullatim levi sudore sub imperium venere Romanum: primo tentatae per Fulvium, deinde praeliis parvis quassatae per Sextium, ad ultimum per Fabium Maximum domitae... Certains modernes ont emboîlé le pas, par exemple Jullian(v. plus haut, n. 5l, à la fin): on doit revenir aujourd'hui, croyons-nous, sur ce jugement; les découvertes des oppida nous font connaître la civilisation avancée de ces petits royaumes indigènes, rabaisser l'importance de Marseille, et mesurer les difficultés que Rome a continué d'avoir au delà des Alpes jusqu'à la fin de l'ère antique. Julliax (III, p. 17, n. 1) a fait juslement remarquer qu'en 121, les 30.000 hommes que StraBo: (IV, 1, 11) prète à l'armée de Gaule constituaient la principale force militaire du monde romain. 